\title{
CALCEOLARIA CALEUANA M.MUÑOZ ET MOREIRA NOM. NOV., NUEVO NOMBRE PARA LA ESPECIE ENDÉMICA CHILENA CALCEOLARIA ESPINOSAE M.MUÑOZ ET MOREIRA (CALCEOLARIACEAE)
}

\author{
CALCEOLARIA CALEUANA M.MUÑOZ ET MOREIRA NOM. NOV., NEW NAME \\ FOR THE CHILEAN ENDEMIC SPECIES CALCEOLARIA ESPINOSAE M.MUÑOZ \\ ET MOREIRA (CALCEOLARIACEAE)
}

\author{
Mélica Muñoz-Schick ${ }^{1} \&$ Andrés Moreira-Muñoz ${ }^{2}$ \\ ${ }^{1}$ Museo Nacional de Historia Natural, Casilla 787, Santiago, Chile; ${ }^{2}$ Instituto de Geografía, \\ Pontificia Universidad Católica de Chile. \\ mmunoz@mnhn.cl
}

\section{ABSTRACT}

A new name for Calceolaria espinosae M.Muñoz et Moreira, non Pennell, is proposed.

En Gayana Botánica 65(1), 2008, los autores describimos una especie de Calceolaria nombrándola en honor del botánico Marcial Espinosa $(C$. espinosae) quien la había recolectado por primera vez en noviembre 1933, en Montenegro, Región Metropolitana. Dicho autor anteriormente la había nombrado en honor a Carlos Reiche, pero sin descripción, por lo tanto era un nomen nudum.

Sin embargo, hemos confirmado que el nombre propuesto por nosotros es ilegítimo, ya que existe con anterioridad Calceolaria espinosae Pennell, en Proc. Acad. Nat. Sci. Philadelphia 103: 170, fig. 26. 1951 (International Plant Names Index, 2009). Dicha especie fue nombrada en honor a Reinaldo Espinosa, quien recolectara la especie en Argelia, provincia de Loja, Ecuador, en junio 1946 (Pennell 1951).

Proponemos entonces el nuevo nombre Calceolaria caleuana M.Muñoz et Moreira nom. nov. El epíteto específico se refiere a la localidad de Caleu, donde fue recolectado el tipo.

Sinónimo: Calceolaria espinosae M.Muñoz et Moreira, Gayana Bot. 65(1): 111-114, 2008. TIPO: Chile, Región Metropolitana, Prov. Chacabuco, Caleu, cumbre Cerro El Roble, 32 $2^{\circ} 59^{\prime} \mathrm{S}, 71^{\circ} 01^{\prime} \mathrm{W}, 2.222 \mathrm{~m}$ s.n.m., A. Moreira 859, 31-XII-2002 (Holotipo SGO 150135).

La especie habita cumbres al norte de Pedernales (V Región de Valparaíso) y en Montenegro y cima del cerro El Roble, Caleu (Región Metropolitana).

\section{AGRADECIMIENTOS}

A Nicolás García quien nos hizo notar esta sinonimia.

\section{BIBLIOGRAFIA}

EspinosA, M. 1933 (publ. 1934). Sociedad Chilena de Historia Natural, Sesión ordinaria, en 15 de Noviembre de 1933. Revista Chilena de Historia Natural 37: 312-314.

International Plant Names Index. 2009. http://www. us.ipni.org/index.html

MuÑoz-SchICK M. \& A. MOREIRA-MuÑoz. 2008. Redescubrimiento de una especie de Calceolaria (Calceolariaceae). Gayana Botánica 65 (1): 111-114.

Pennell, F.W. 1951. The genus Calceolaria in Ecuador, Colombia and Venezuela. Proc. Acad. Nat. Sci. Philadelphia 103: 85-196, 2 lám. 